\title{
MODELO DE NICHO FUNDAMENTAL PARA Coryphantha chihuahuensis (CACTACEAE) EN EL ESTADO DE CHIHUAHUA, MÉXICO
}

\section{FUNDAMENTAL NICHE MODEL OF Coryphantha chihuahuensis (CACTACEAE) IN THE STATE OF CHIHUAHUA, MÉXICO}

\author{
Irma Ibarra-Díaz Velarde ${ }^{1}$, Toutcha Lebgue-Keleng ${ }^{2}$, Oscar Viramontes-Olivas ${ }^{3}$, Ignacio Reyes-Cortes ${ }^{4}$, Juan A. \\ Ortega-Gutierrez ${ }^{2}$ y Carlos Morales-Nieto ${ }^{2}$
}

\begin{abstract}
Resumen
La familia Cactaceae se distingue por su morfología y anatomía. Que le permite soportar épocas prolongadas de sequías y alta radiación solar. Para algunas de estas especies se carece de información relacionada con su distribución, estatus y nicho fundamental. Es el caso de la cactácea Coryphantha chihuahuensis, reportada en el estado de Chihuahua y en el Desierto Chihuahuense en México. El objetivo de este trabajo fue determinar el nicho fundamental de C. chihuahuensis en el estado de Chihuahua. Con ello se muestran las áreas probables y adecuadas para el desarrollo de la especie. Se georeferenciaron en campo 20 sitios de presencia de la especie. Para determinar su nicho fundamental se utilizaron tres modelos con distintas combinaciones de variables (bioclimáticas, altitud, edafología, usos de suelo y vegetación). El algoritmo utilizado fue MaxEnt (máxima entropía). Los resultados indicaron que el modelo con menor error fue el que utilizó todas las variables, con un valor de efectividad de $99 \%$ y una desviación estándar de 0.001 . El modelo muestra el nicho fundamental de la especie al centro y sur del estado, con una probabilidad de ocurrencia del 70 al 100\% al centro; mientras que los valores más bajos fueron al sur-centro y al noreste del estado con probabilidad de presencia del 30 al 50\%. El modelo obtenido para determinar el nicho fundamental para $C$. chihuahuense es puntual, ya que muestra las áreas probables y adecuadas para la especie. Esto ayudará a la protección, conservación y recuperación de la misma.
\end{abstract}

Palabras clave: máxima entropía, ocurrencia, endémicas, georeferencia, variables bioclimáticas, nicho fundamental.

\begin{abstract}
The Cactaceae family is distinguished by its morphology and anatomy that allows it to withstand prolonged periods of drought and high solar radiation. For some of its species there is a lack of basic information regarding distribution, status and fundamental niche. This is the case of Coryphantha chihuahuensis, reported in the Chihuahua state in the Chihuahuan Desert region. The aim of this study was to determine the fundamental niche of Coryphantha chihuahuensis in the state of Chihuahua, displaying possible and suitable areas in which the species could develop. During fieldwork 20 georeferenced sites with species occurrence were recorded. The species fundamental niche was determined using three models with different combinations of variables (bioclimatic variables, altitude, soil, land use and vegetation). The algorithm used was MaxEnt (maximum entropy). The results indicate that the model with the lowest error was the model that contained all variables, with an effectiveness value of $99 \%$ and a standard deviation of 0.001 . The model places the fundamental niche of the species in the center and south of the state, with a probability of occurrence of 70 to $100 \%$ at the center; while the lowest values were south-central and northeast of the state with the probability of presence of 30 to $50 \%$. The obtained model used to determine the fundamental niche for C. Chihuahua is timely as it shows probable and appropriate areas for the species. This will help in the protection, conservation and recovery of the species.
\end{abstract}

Key words: maximum entropy, occurrence localities, endemic, georeference, environmental variables, fundamental niche. 


\section{Introducción.}

La familia Cactaceae es nativa del continente americano (Contreras-Medina et al., 2010; Nyffeler \& Eggli, 2010; Lebgue-Keleng et al., 2014). Por tener una morfología y anatomía que les permiten soportar épocas prolongadas de sequias y alta radiación solar, la mayoría de sus especies están adaptadas a regiones áridas y semiáridas. La familia está constituida por 110 a 122 géneros y entre 1,500 y 1,600 especies (Lebgue-Keleng et al., 2011). Estudios efectuados por Hernández et al. (2010), Prado et al. (2010) y LebgueKeleng et al. (2014) reconocieron que México está identificado como el país con la mayor diversidad de cactáceas. El Desierto Chihuahuense es considerado una de las regiones de mayor diversidad de cactáceas, con 329 especies y 39 géneros; de estos taxones, 70\% de las especies y $43 \%$ de los géneros fueron reportados como endémicos (Hernández et al., 2010). Lebgue-Keleng et al. (2014) mencionaron que el estado de Chihuahua alberga el $16 \%$ de esta diversidad endémica.

Coryphantha chihuahuensis Lem., también identificada como Escobaria chihuahuensis Britton \& Rose, es una especie poco estudiada. El Herbario del Jardín Botánico de Missouri (MO) y el Herbario Royal Botanic Garden (MEL) del Reino Unido realizaron una descripción de la especie y la ubicaron sólo en el estado de Chihuahua, México, en las colinas rocosas cercanas a la ciudad de Chihuahua. Asimismo, Estrada-Castillo \& Villarreal-Quintanilla (2010) reportaron esta especie en el centro del estado de Chihuahua.

Los modelos de distribución geográfica son importantes para aplicaciones ecológicas y de conservación biológica (Phillips \& Dudik, 2008; Ibarra-Montoya et al., 2012). El propósito de estos modelos es predecir las condiciones ambientales idóneas de las especies como una función de las variables ambientales (Phillips et al., 2006). Los nichos ecológicos se basan en modelos que tienen la capacidad de proyectar patrones de distribución geográfica, mediante los registros puntuales de la especie en cuestión (Milesi \& López de Casevave, 2005; Mercado \& Wallace, 2010; Ibarra-Montoya et al., 2012; Pérez-García \& Liria, 2013). El nicho fundamental señala el rango multivariado de las tolerancias fisiológicas a variables climáticas principalmente, dentro del cual una especie tendrá tasas positivas de crecimiento. A ésta se le denomina distribución potencial (Mercado \& Wallace, 2010; Soberón \& Peterson, 2011).

En la actualidad existen programas computacionales para determinar el nicho fundamental de una especie (Phillips et al., 2006; Mercado \& Wallace, 2010; Pliscoff \& Fuentes-Castillo, 2011). Para la modelación de nichos, Contreras-Medina et al. (2010), Ibarra-Montoya et al. (2012) y Pliscoff \& Fuentes-Castillo (2011) mencionaron que el programa
MaxEnt (máxima entropía) (Phillips et al., 2006), ha sido evaluado y probado por distintos investigadores, catalogándolo como uno de los mejores modeladores.

Considerando la importancia ecológica de las cactáceas y sus características biológicas (como bajas tasas de crecimiento y bajas probabilidades de establecimiento) que las hacen más vulnerables al disturbio, es necesario proteger las pocas poblaciones existentes y multiplicarlas para su restauración en zonas con potencial de expansión, aumentando la necesidad y el compromiso de conocer más sobre los factores que controlan la distribución y abundancia de C. chihuahuensis y otras especies de cactáceas.

Dada la información limitada de $C$. chihuahuensis, no se conocen aspectos precisos de su distribución geográfica y estatus ecológico. Por lo anterior, el objetivo de la presente investigación fue determinar el nicho fundamental de $C$. chihuahuensis para generar información que pueda ser utilizada en el diseño de planos para la protección, conservación y recuperación de la especie.

\section{Materiales y métodos.}

Coryphantha chihuahuensis es una especie colectada por primera vez por Palmer en el año 1908 en montañas a $20 \mathrm{~km}$ de la ciudad de Chihuahua, Chih. (Bravo-Hollis \& Sanchez- Mejorada 1991). Lebgue \& Quintana (2014) mencionaron que la especie cuenta con una posible distribución restringida, y la ubican en los municipios de Chihuahua y Rosales, sugiriendo estudios que aporten información más precisa. Coryphantha chihuahuensis es una planta solitaria a cespitosa, con tallos cilíndricos de 7 a $9 \mathrm{~cm}$ de alto y de 4 a $5 \mathrm{~cm}$ de diámetro, con tubérculos. Inflorescencia en tubérculos superiores cerca del ápice en color rosa. La especie se desarrolla en un hábitat con tipo de vegetación de Matorral Xerófilo Micrófilo y Matorral Xerófilo Rosetifolio, en asociación con vegetación de climas áridos y semiáridos, con plantas suculentas y áfilas. Se localiza en elevaciones de 1,200 a 1,700 msnm (Bravo-Hollis y Sanchez- Mejorada 1991; Lebgue \& Quintana, 2014).

El trabajo de campo se realizó en los meses de julio y agosto del 2013 y consistió en localizar puntos de presencia - ausencia de $C$. chihuahuensis. Se registraron 56 puntos de muestreo, de los cuales solo en 20 se localizó la especie. Wisz et al. (2008) mencionaron que para una especie poco conocida, los modelos con tamaños de muestra por debajo de 30 puntos pueden producir resultados útiles. Los mismos autores expusieron, que para modelos exploratorios con tamaños de muestra entre 10 y 30 registros, MaxEnt es uno de los programas más adecuados. El estudio se realizó en las colinas rocosas de los municipios de Chihuahua, Aldama, Coyame del Sotol, Ojinaga, Aquiles Serdán, Rosales, Meoqui, Delicias, Satevó, Valle de Zaragoza, Hidalgo del Parral en el 
estado de Chihuahua (Figura 1). La selección de estos municipios se basó en el estudio de Estrada-Castillo \& Villarreal-Quintanilla (2010), quienes especificaron que la especie se localiza en la parte central del estado de Chihuahua. También, se tomó como referencia el tipo de vegetación de las cartas de INEGI de tipos de vegetación y uso de suelo para el estado de Chihuahua escala 1:250,000. Lo anterior se consideró para planear los recorridos de campo. El punto de referencia fue la ciudad de Chihuahua ubicada a una latitud norte de $28^{\circ} 38^{\prime} 07^{\prime}$ y una longitud oeste de $106^{\circ}$ 05' 20". Se tomaron como ejes las carreteras Chihuahua - Ojinaga, Chihuahua - Parral, Chihuahua - Camargo y Chihuahua - Cd. Juárez. Sobre estos ejes se recorrieron, caminos vecinales, brechas y veredas para detectar cerros y colinas rocosas. Estas áreas fueron examinadas para determinar ausencia 0 presencia de la especie y se georeferenciaron (longitud, latitud y altitud). Se estableció una distancia mínima de 30 a $50 \mathrm{~km}$ entre los puntos de observación; sin embargo, en algunas ocasiones el trayecto era mayor, ya que no se encontraron las características deseadas. Para corroborar la ubicación de cada punto georreferenciado se utilizó Google Earth versión 7.1.1.1888, recomendado por Potere (2008) y Pérez-García \& Liria (2013) como una fuente de datos de alta resolución y precisión (Figura 2).

Para determinar el nicho fundamental de Coryphantha chihuahuensis se utilizó el programa MaxEnt 3.3.3, una aproximación estadística basada en un algoritmo de máxima entropía (Phillips et al., 2006; Phillips \& Dudik, 2008), con la capacidad de predecir o inferir sobre información incompleta. Este programa genera proyecciones espaciales de distribución potencial de especies a partir de nueve datos de presencia, aunque lo recomendado son cercanos a 20 (Phillips et al. 2006; Wisz et al., 2008; Mercado \& Wallace, 2010; Pliscoff \& Fuentes-

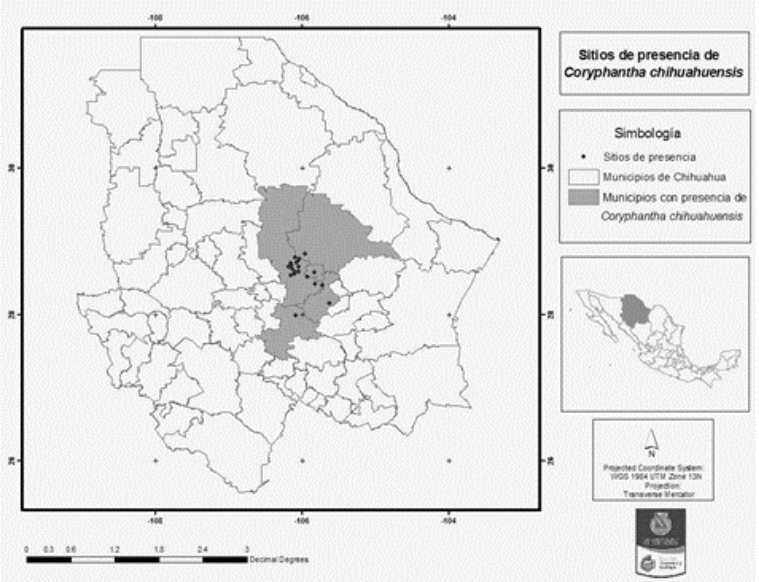

Figura 1. Ubicación de la zona de estudio y puntos de presencia de Coryphantha chihuahuensis en el estado y municipios de Chihuahua.
Tabla 1. Variables bioclimáticas utilizadas parar la construcción de los modelos.

\begin{tabular}{|c|c|}
\hline Clave & Descripción \\
\hline Bio 1 & Temperatura media anual \\
\hline Bio 2 & $\begin{array}{l}\text { Intervalo del promedio de temperatura diurna } \\
\text { (media mensual (temperatura máxima- } \\
\text { temperatura media)) }\end{array}$ \\
\hline
\end{tabular}

Bio 3 Isoterma $(* 100)$

Bio 4 Estacionalidad de la temperatura (desviación standard*100)

Bio 5 Temperatura máxima del mes más caliente,

Bio 6 Temperatura mínima del mes más frío

Bio 7 Intervalo de temperatura anual

Bio 8 Temperatura promedio del trimestre más húmedo

Bio 9 Temperatura promedio del trimestre más seco

Bio 10 Temperatura promedio del trimestre más cálido

Bio 11 Temperatura promedio del trimestre más frío

Bio 12 Precipitación anual

Bio 13 Precipitación del mes más húmedo

Bio 14 Precipitación del mes más seco

Bio 15 Estacionalidad de la precipitación coeficiente de variación)

Bio 16 Precipitación del trimestre más húmedo

Bio 17 Precipitación del trimestre más seco

Bio 18 Precipitación del trimestre más cálido

Bio 19 Precipitación del trimestre más frío

(Pérez-García \& Liria, 2013).

Castillo, 2011). La distribución de la especie se definió por probabilidades mediante la salida logística propia del programa, donde se muestra la probabilidad de que la especie esté presente de acuerdo a un porcentaje del 0 a $100 \%$. Para correr el programa se utilizaron datos de presencia y variables ambientales cualitativas o cuantitativas (Phillips et al. 2006; Mercado \& Wallace, 2010; Pérez-García \& Liria, 2013).

Se utilizaron 19 variables bioclimáticas (Tabla 1), altitud, edafología, usos de suelo y vegetación. La utilización de estas variables fue para estimar las más representativas a la especie, y las condiciones en las que se pudiera ubicar. Los valores mensuales y anuales de temperatura y precipitación fueron tomadas
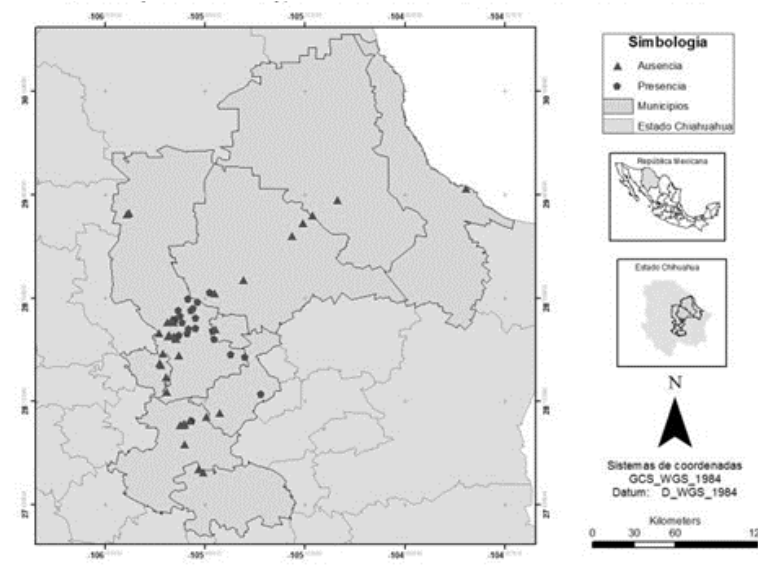

Figura 2. Presencia y ausencia de Coryphantha chihuahuensis en el estado de Chihuahua y sus municipios. 
Tabla 2. Puntos de presencia de Coryphantha chihuahuensis. Estudio de campo (julio y agosto del 2013).

\begin{tabular}{rlrc}
\hline ID & Lugar & $\mathrm{X}$ & $\mathrm{Y}$ \\
\hline 1 & Camino a Horcasitas & $28^{\circ} 25^{\prime} 08^{\prime \prime}$ & $105^{\circ} 49^{\prime} 18^{\prime \prime}$ \\
2 & Cerca Bachiniva & $28^{\circ} 23^{\prime} 59^{\prime \prime}$ & $105^{\circ} 43^{\prime} 47^{\prime \prime}$ \\
3 & Grutas Nombre de Dios & $28^{\circ} 42^{\prime} 20^{\prime \prime}$ & $106^{\circ} 04^{\prime} 48^{\prime \prime}$ \\
4 & Granjas universidad & $28^{\circ} 33^{\prime} 13^{\prime \prime}$ & $106^{\circ} 06^{\prime} 19^{\prime \prime}$ \\
5 & Cerro San Sebastián & $28^{\circ} 42^{\prime} 18^{\prime \prime}$ & $106^{\circ} 09^{\prime} 41^{\prime \prime}$ \\
6 & Libra Delicias- Juárez & $28^{\circ} 46^{\prime} 53^{\prime \prime}$ & $106^{\circ} 05^{\prime} 54^{\prime \prime}$ \\
7 & Nombre de Dios feria Santa Rita & $28^{\circ} 39^{\prime} 10^{\prime \prime}$ & $106^{\circ} 02^{\prime} 55^{\prime \prime}$ \\
8 & Presa las vírgenes & $28^{\circ} 09^{\prime} 18^{\prime \prime}$ & $105^{\circ} 37^{\prime} 30^{\prime \prime}$ \\
9 & Carretera Delicias - Satevo & $27^{\circ} 59^{\prime} 08^{\prime \prime}$ & $106^{\circ} 05^{\prime} 15^{\prime \prime}$ \\
10 & Delicias - Chih km. 201 & $28^{\circ} 30^{\prime} 53^{\prime \prime}$ & $105^{\circ} 55^{\prime} 47^{\prime \prime}$ \\
11 & Boulevard a Almada & $28^{\circ} 34^{\prime} 52^{\prime \prime}$ & $106^{\circ} 05^{\prime} 49^{\prime \prime}$ \\
12 & Cerro grande & $28^{\circ} 35^{\prime} 12^{\prime \prime}$ & $106^{\circ} 02^{\prime} 57^{\prime \prime}$ \\
13 & Municipio Aquiles Sedán & $28^{\circ} 34^{\prime} 14^{\prime \prime}$ & $105^{\circ} 50^{\prime} 04^{\prime \prime}$ \\
14 & Ejido Labor de Terrazas & $28^{\circ} 32^{\prime} 21^{\prime \prime}$ & $106^{\circ} 09^{\prime} 24^{\prime \prime}$ \\
15 & Prolongación Francisco Villa & $28^{\circ} 40^{\prime} 04^{\prime \prime}$ & $106^{\circ} 08^{\prime} 53^{\prime \prime}$ \\
16 & Pista aviones prolongación Cantera & $28^{\circ} 38^{\prime} 57^{\prime \prime}$ & $106^{\circ} 11^{\prime} 02^{\prime \prime}$ \\
17 & Cerro atrás CIMA & $28^{\circ} 37^{\prime} 24^{\prime \prime}$ & $106^{\circ} 08^{\prime} 10^{\prime \prime}$ \\
18 & Carretera Aldama Autódromo & $28^{\circ} 49^{\prime} 21^{\prime \prime}$ & $105^{\circ} 57^{\prime} 34^{\prime \prime}$ \\
19 & Carretera Aldama libramiento Juárez-Delicias & $28^{\circ} 45^{\prime} 24^{\prime \prime}$ & $106^{\circ} 02^{\prime} 16^{\prime \prime}$ \\
20 & Centro Cerro Nombre de Dios & $28^{\circ} 42^{\prime} 57^{\prime \prime}$ & $106^{\circ} 04^{\prime} 06^{\prime \prime}$ \\
\hline
\end{tabular}

“acumulativo” indica por pixel (1 x $1 \mathrm{~km}$ ) el hábitat idóneo para la especie. Para estimar la presencia de la especie se utilizan rangos del 0 al 100 , donde 100 y 0 es la mayor y menor probabilidad de presencia, respectivamente (Phillips et al., 2006; IbarraMontoya et al., 2012).

Se ingresaron al programa 20 puntos de presencia (Tabla 2) junto con las distintas combinaciones de variables. Las primeras variables a utilizar fueron las bioclimáticas, llamado modelo 1. Para el modelo 2 fueron las variables bioclimáticas en combinación con la altitud. En el modelo 3 se utilizaron las variables bioclimáticas, altitud, edafología, usos de suelo y vegetación.

El análisis estadístico se realizó por medio del programa MaxEnt. El cual se basa en un sistema estadístico de $\mathrm{P}$ probabilidad. Su análisis se basa en la función probabilística $\mathrm{P}$ sobre un conjunto $\mathrm{x}$, o sea, puntos de registros. El modelo $\mathrm{P}$ se construye mediante un conjunto de restricciones derivadas del promedio empírico de ocurrencia, asociados a variables ambientales conocidas $\mathrm{f}(\mathrm{v})$. Esto basado en el índice de Shannon, donde de las posibles combinaciones de funciones se utiliza la que minimiza la función de entropía (Phillips et al., 2006).

Se obtuvieron las tres salidas logísticas con los modelos del distribución probable de $C$. chihuahuensis. Estos modelos fueron incorporados a un Sistema de Información Geográfica (SIG) con la finalidad de generar mapas de consenso de valores por pixel (1 X $1 \mathrm{~km}$ ). Para ello se determinó un umbral de corte al $30 \%$ de probabilidad, con el $10 \%$ de las poblaciones por debajo, resultado de MaxEnt. Siendo este porcentaje el más bajo dentro de los tres modelos y con el fin de uniformizar los resultados. Los rangos utilizados fueron: de 100 a $85 \%, 70$ a $85 \%$, 50 a $70 \%$, 50 a $30 \%$ y de 30 a $0 \%$, donde $100 \%$ es máxima probabilidad y $0 \%$ es ausencia. Wallace, 2010). El jacknife, otro de los resultados del programa, calcula la contribución de cada variable al modelo. Esto evidencia los principales requerimientos ambientales de la especie y por tanto, determinará la posible variable significativa para establecer la distribución potencial. La salida del programa

Tabla 3. Probabilidad de presencia de Coryphantha chihuahuensis en el estado de Chihuahua, bajo tres modelos de nicho fundamental.

\begin{tabular}{|c|c|c|c|c|c|c|c|c|c|}
\hline \multirow[t]{2}{*}{$\begin{array}{l}\text { Probabilidad } \\
\text { de presencia }\end{array}$} & \multirow[t]{2}{*}{$\begin{array}{c}\text { Modelo } 1 \\
\text { Área ha }\end{array}$} & \multicolumn{2}{|c|}{$\begin{array}{l}\text { Sitios de } \\
\text { muestreo }\end{array}$} & \multirow{2}{*}{$\begin{array}{c}\text { Modelo } \\
2 \\
\text { Área ha }\end{array}$} & \multicolumn{2}{|c|}{$\begin{array}{l}\text { Sitios de } \\
\text { muestreo }\end{array}$} & \multirow[t]{2}{*}{$\begin{array}{c}\text { Modelo } 3 \\
\text { Área ha }\end{array}$} & \multicolumn{2}{|c|}{$\begin{array}{l}\text { Sitios de } \\
\text { muestreo }\end{array}$} \\
\hline & & $\mathrm{P}$ & A & & $\mathrm{P}$ & A & & $\mathrm{P}$ & A \\
\hline 85 а $100 \%$ & 56 & 10 & 1 & 82 & 6 & 2 & 51 & 13 & 0 \\
\hline 70 а $85 \%$ & 105 & 4 & 4 & 140 & 6 & 2 & 82 & 6 & 4 \\
\hline 50 а $70 \%$ & 171 & 3 & 4 & 386 & 8 & 10 & 166 & 1 & 4 \\
\hline 30 а $50 \%$ & 459 & 3 & 3 & 520 & 0 & 4 & 372 & 0 & 8 \\
\hline 0 а $30 \%$ & 27,255 & 0 & 24 & 26,918 & 0 & 18 & 27,375 & 0 & 20 \\
\hline
\end{tabular}




\section{Resultados y discusión.}

Se cuestiona si la especie es endémica del estado de Chihuahua, debido a que Hernández et al., (2004) la mencionaron en un listado de especies en el Desierto Chihuahuense en los estados de Chihuahua y Durango. Los mismos autores reportaron a Coryphantha chihuahuensis como Escobaria chihuahuensis. Dicho estudio se basa en un análisis literario, más no existe una corroboración en campo. Además de este estudio no se encontró otro registro de la especie fuera del estado de Chihuahua.

Los tres modelos resultantes son similares, señalan una distribución probable de Coryphantha chihuahuensis al centro y sur del estado de Chihuahua principalmente.

El modelo 1 marca una máxima probabilidad de presencia en torno a la ciudad de Chihuahua, seguida radialmente por rangos de menor magnitud que se extienden al sur (Figura 3A). Los 20 puntos de presencia quedan dentro de estos rangos y suman un área de 761 hectáreas. El modelo 2 muestra un comportamiento menos delimitado que el modelo anterior (Figura 3B). Este señala la probabilidad máxima probable al entorno de la ciudad de Chihuahua, cubre mayor área (1,128 ha.) y se extiende al sur de la misma. El tercer modelo señala una distribución más puntual que los demás modelos (Figura 3C). Aquí se ubican 13 puntos de presencia y cero de ausencia dentro del máximo rango, con un área potencial de 671 hectáreas (Tabla 3). Los tres modelos predicen y delimitan la distribución potencial de la especie al estado de Chihuahua, corroborando las observaciones hechas en campo.

Los resultados AUC muestran una buena predicción de los tres modelos, con valores $>0.90$; la desviación estándar del modelo 1 y 3 es de 0.001 y para el modelo 2 es 0.002 . La variable que aporta más a los tres modelos, resultado de jacknife, es la variable denominada Bio 2 (intervalo del promedio de temperatura diurna media mensual), una de las 19 variables bioclimáticas, por lo que se concluye que las variables bioclimáticas por si solas son capaces de delimitar la distribución actual de C. chihuahuensis, siendo esta variable en particular un elemento importante en la distribución y persistencia de sus poblaciones.

\section{Conclusiones.}

Los tres modelos muestran que la probabilidad de distribución de Coryphantha chihuahuensis se extiende del centro del estado de Chihuahua en forma radial y se incrementa al sur del mismo. Con algunas zonas aisladas al noreste y sureste del estado.

Para estimar el nicho fundamental de $C$. chihuahuensis, los tres modelos tuvieron comportamientos similares y una validación a uno. Sin embargo, el modelo tres presentó menor error, ya que contiene mayor número de puntos de presencia (13 de

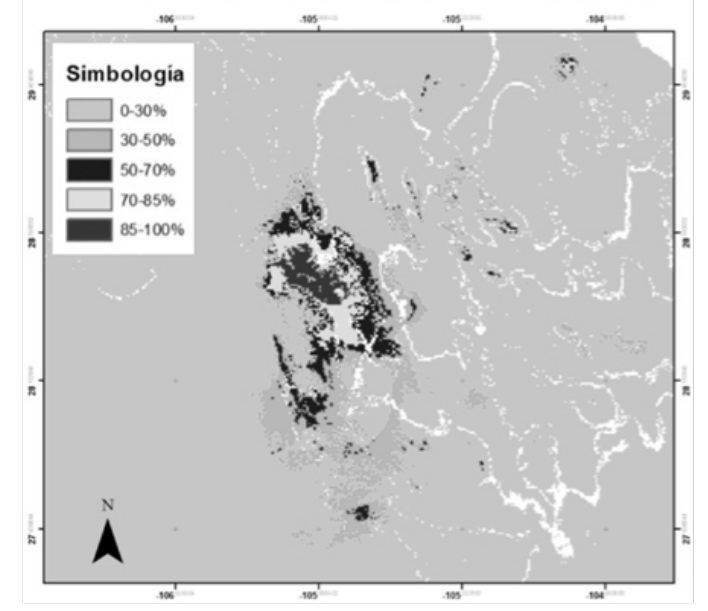

Figura 3A: Modelo 1. Variables bioclimáticas

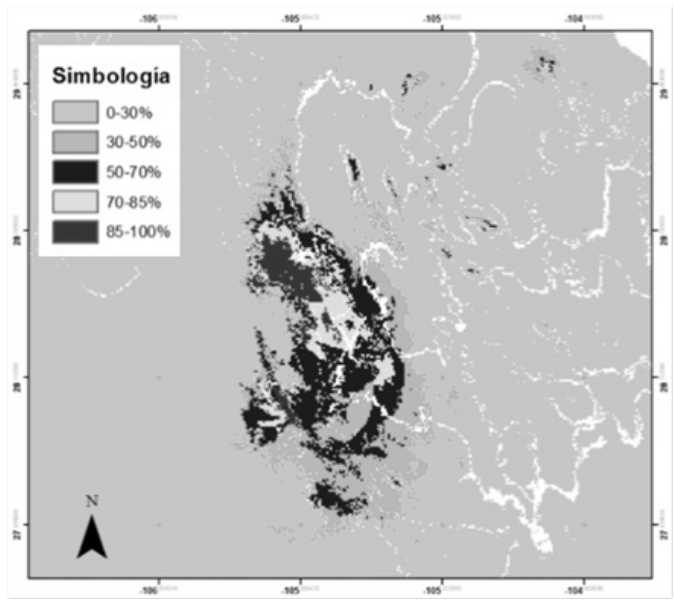

Figura 3B: Modelo 2. Variables bioclimáticas altura

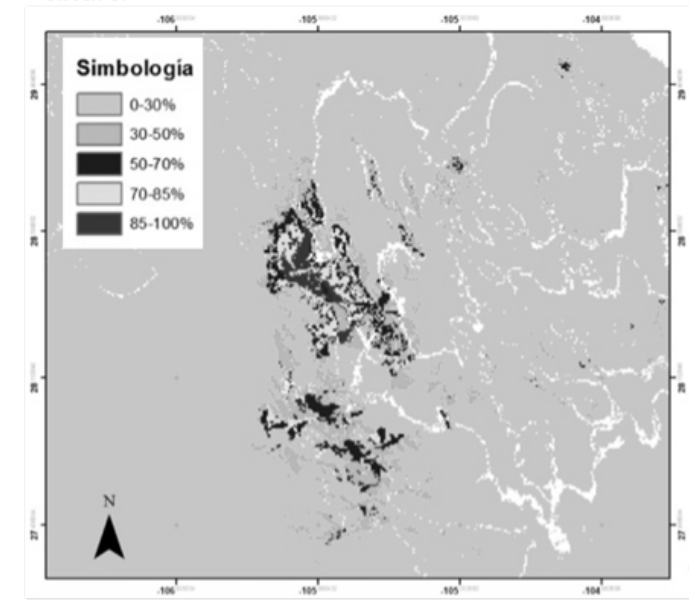

Figura 3C: Modelo 3. Variables bioclimáticas, altitud, edafología, usos de suelo y vegetación

Figura 3. Modelos de nicho fundamental de Coryphantha chihuahuensis en el estado de Chihuahua. Probabilidad de ocurrencia de la especie por rangos donde $100 \%$ es máxima presencia y $0 \%$ ausencia. 
20), dentro de la mayor probabilidad de presencia (70 a $100 \%)$.

El programa MaxEnt es una herramienta útil para modelar la distribución de especies como $C$. chihuahuensis, ya que facilita y agiliza resultados confiables para determinar el nicho fundamental.

El modelo obtenido para determinar el nicho fundamental para $C$. chihuahuense en el estado de Chihuahua es puntual, ya que muestra las áreas probables y adecuadas para la especie. No obstante, una de las principales amenazas para esta especie podría ser su cercanía a los desarrollos urbanos.

Los resultados son relevantes debido a que no se contaba con datos de ubicación ni del nicho fundamental de esta cactácea. Esta información puede ser de utilidad para la protección, conservación y recuperación de esta especie. Debe destacarse que a pesar de que la distribución actual observada se ajusta bien al modelo, queda la limitante de incluir variables de pendiente y exposición al modelo, que quizás pudieran aportar y generar un nicho fundamental todavía más preciso.

Por otro lado, se recomienda ampliar el estudio, mediante visitas de campo a sitios con tipos de vegetación de Matorral Xerófilo Micrófilo y Matorral Xerófilo Rosetifolio fuera del estado de Chihuahua. Para corroborar la ausencia de la especie en más estados y poder confirmar el posible endemismo dentro del estado de Chihuahua o del Desierto Chihuahuense. Además de ampliar la información de la especie mediante un análisis de asociación vegetativa y edáfica. Así como, realizar un estudio demográfico completo que ayudaría a determinar si se encuentra en alguna categoría de peligro o vulnerabilidad.

\section{Agradecimientos.}

Este estudio no hubiera sido posible sin el apoyo financiero de Conacyt, por la beca recibida en los estudios de posgrado. Al Gobierno del Estado de Chihuahua y a la Facultad de Zootecnia y Ecología de la Universidad Autónoma de Chihuahua, por proveer los medios necesarios para los recorridos de campo y la toma de datos.

\section{Literatura citada.}

Bravo-Hollis H. \& Sánchez-Mejorada H. 1991. Las cactáceas de México vol. II. Editor Universidad Nacional Autónoma de México. México.

Contreras-Medina R., Luna-Vega I. \& Ríos-Muñoz C. 2010. Distribución de Taxus globosa (Taxaceae) en México: Modelos ecológicos de nichos, efectos del cambio del uso de suelo y conservación. Revista chilena de historia natural. 83:421-433.

Estrada-Castillo E. \& Villarreal- Quintanilla J.A. 2010 Flora del centro del estado de Chihuahua, México. Acta Botánica Mexicana. 92:51-118.
Hernández H., Gómez-Hinostrosa C. \& Goettsch B. 2004. Checklist of Chihuahuan Desert Cactaceae. Harvard Papers in Botany. 9: 51-68.

Hernández H., Gómez-Hinojosa C. \& Hoffman G. 2010. ¿Es la rareza geográfica frecuente entre las cactáceas del Desierto Chihuahuense? Revista mexicana de Biodiversidad. 81:183-175.

Ibarra-Montoya J., Rangel-Peraza G., González-Farias F., Anda J., Martínez-Meyer E. \& Macias-Cuellar H. 2012. Uso del modelado de nicho ecológico como una herramienta para predecir la distribución potencial de Microcystis sp. (cianobacteria) en la Presa Hidroeléctrica de Aguamilpa, Nayarit, México. Ambiente \& Agua. 7(1): 218-234.

Lebgue-Keleng T., Viramontes-Olivas O., Soto-Cruz R., Quiñonez-Martínez M., Balderrama-Castañeda S. \& Aviña-Dominguez Y. 2011. Cactáceas endémicas y raras del estado de Chihuahua, México. Tecnociencia Chihuahua. 5(1):27-33.

Lebgue-Keleng T. Aviña-Dominguez Y., Soto-Cruz R., Quiñonez-Martínez M., Cortes-Palacios L., MelgozaCastillo A., Morales-Nieto C., Vélez-Sanchez-Verin C. \& Balderrama-Castañeda S. 2014. Soil mineralogy and its relationship with cacti species in southeastern Chihuahua, México. Ecología Aplicada. 13:205-209.

Lebgue \& Quintana. 2014. Cactáceas de Chihuahua. Segunda edición. Chihuahua. México. Proveedor Integracional y Servicios Ambientales, Gustavo Quintana Martínez, Instituto Chihuahuense de la Cultura. Chihuahua, México.

Mercado N. \& Wallace R. 2010. Distribución de primates en Bolivia y áreas prioritarias para su conservación. Tropical Conservation Science. 3:200-217.

Milesi F. A. \& López de Casevave J. 2005. El concepto de nicho en Ecología aplicada: del nicho al hecho hay mucho trecho. Ecología Austral. 15:131-148.

Nyffeler R. \& Eggli U. 2010. A farewell to dated ideas and concepts: molecular phylogenetics and a revised suprageneric classification of the family Cactaceae. Shumannia. 6:109-149.

Pérez-García B. \& Liria J. 2013. Modelos de nicho ecológico fundamental para especie del género Thraulodes (Ephemeroptera: Leptophlebiidae: Atalophleiinae). Revista Mexicana de Biodiversidad. 84: 600-611.

Peterson A. T., Papes M. \& Soberón J. 2008. Rethinking receiver operating characteristic analysis applications in ecological niche modeling. Ecological Modelling. 213:63-72.

Phillips S., Anderson R. \& Eschaoure R. 2006. Maximum entropy modeling of species geographic distributions. Ecological Modelling. 190:231-259.

Phillips S. \& Dudik M. 2008. Modeling of species distribution with Maxent: new extensions and a comprehensive evaluation. Ecography. 31:161-175.

Pliscoff P. \& Fuentes-Castillo T. 2011. Modelación de la distribución de especies y ecosistemas en el tiempo y en el espacio: una revisión de las nuevas herramientas y enfoques disponibles. Geografía Norte Grande. 48:6179.

Potere D. 2008. Horizontal positional accuracy of Google Earth's high - resolution imagery archive. Sensors. 8:7973-7981. 
Prado A., Hawkins J., Yesson C. \& Bárcenas R. T. 2010. Multiple diversity measures to identify complementary conservation areas for Baja California peninsular cacti. Biological Conservation. 143:1510-1520.

Soberón J. \& Peterson T. 2011. Ecological niche shifts and environmental space anisotropy: a cautionary note. Revista Mexicana de Biodiversidad. 82:1348-1355.
Wisz M.S., Hijmans R.J., Li J., Peterson A.T., Graham, A. Guisan C.H. \& NCEAS Predicting Species Distributions Working Group. 2008. Effects of sample size on the performance of species distribution models. Diversity and Distributions. 14: 763-773.

${ }^{1}$ Estudiante de la Facultad de Zootecnia y Ecología de la Universidad Autónoma de Chihuahua. Periférico Francisco R. Almada, Km 1, Chihuahua, Chih. México, CP. 31031. kidv5@hotmail.com; 52-614-434 0304.

${ }^{2}$ Profesor-Investigador de la Facultad de Zootecnia y Ecología de la Universidad Autónoma de Chihuahua. Periférico Francisco R. Almada, Km 1, Chihuahua, Chih. México, CP. 31031. tlebgue@uach.mx; jortega@uach.mx; cnieto@uach.mx; 52-614-434 0304.

3 Profesor-Investigador de la Facultad de Contaduría y Administración de la Universidad Autónoma de Chihuahua. Circuito Universitario \#1, Nuevo Campus Universitario, Chihuahua, Chih. México, CP. 31125. oviramon@uach.mx; 52-614- 4420030.

4 Profesor-Investigador de la Facultad de Ingeniería de la Universidad Autónoma de Chihuahua. Circuito universitario s/n Campus II, Chihuahua, Chih. México, CP. 31325. iareyes2020@gmail.com; 52-614-442 95 00 . 